\title{
Stimulus Similarity-Contingent Neural Adaptation Can Be Time and Cortical Area Dependent
}

\author{
Bram-Ernst Verhoef, ${ }^{\star}$ Greet Kayaert, ${ }^{\star}$ Edit Franko, Joris Vangeneugden, and Rufin Vogels \\ Laboratorium voor Neuro-en-Psychofysiologie, Katholieke Universiteit Leuven Medical School, BE-3000 Leuven, Belgium
}

\begin{abstract}
Repetition of a stimulus results in decreased responses in many cortical areas. This so-called adaptation or repetition suppression has been used in several human functional magnetic resonance imaging studies to deduce the stimulus selectivity of neuronal populations. We tested in macaque monkeys whether the degree of neural adaptation depends on the similarity between the adapter and test stimulus. To manipulate similarity, we varied stimulus size. We recorded the responses of single neurons to different-sized shapes in inferior temporal (IT) and prefrontal cortical (PFC) areas while the animals were engaged in a size or shape discrimination task. The degree of response adaptation in IT decreased with increasing size differences between the adapter and the test stimuli in both tasks, but the dependence of adaptation on the degree of similarity between the adapter and test stimuli was limited mainly to the early phase of the neural response in IT. PFC neurons showed only weak size-contingent repetition effects, despite strong size selectivity observed with the same stimuli. Thus, based on the repetition effects in PFC, one would have erroneously concluded that PFC shows weak or no size selectivity in such tasks. These findings are relevant for the interpretation of functional magnetic resonance adaptation data: they support the conjecture that the degree of adaptation scales with the similarity between adapter and test stimuli. However, they also show that the temporal evolution of adaptation during the course of the response, and differences in the way individual regions react to stimulus repetition, may complicate the inference of neuronal tuning from functional magnetic resonance adaptation.
\end{abstract}

Key words: inferior temporal cortex; prefrontal cortex; repetition suppression; macaques; size selectivity; fMRI adaptation

\section{Introduction}

Previous studies have demonstrated that stimulus repetition results in decreased activity in visual cortical neurons (GrillSpector et al., 2006; Krekelberg et al., 2006; Kohn, 2007). Recently, there has been an increase in the number of functional magnetic resonance imaging (fMRI) studies that use such "adaptation" or "repetition suppression" to deduce the stimulus selectivity of neuronal populations in humans [functional magnetic resonance adaptation (fMR-A)] (Naccache and Dehaene, 2001; Grill-Spector et al., 2006; Krekelberg et al., 2006). Inferring neural tuning using fMR-A assumes that the degree of adaptation depends on stimulus similarity if the cells of the region under study are selective for these stimuli (Jiang et al., 2006). Single-cell studies in early visual areas have indeed shown that the strength of such adaptation effects depends on the degree of similarity between the test and adapter stimuli (Kohn, 2007). However,

Received July 17, 2008; revised Sept. 3, 2008; accepted Sept. 4, 2008.

This work was supported by Geconcerteerde Onderzoeksacties K.U. Leuven (GOA/2005/18), Excellentiefinanciering K.U. Leuven (EF/05/014), Interuniversitaire Attractiepool Belgium (IUAP P6/29), Geneeskundige Stichting Koningin Elisabeth, Human Frontier Science Program, and the Fund for Scientific Research (FWO) Flanders. G.K. and J.V. are postdoctoral and doctoral fellows, respectively, of the FWO Flanders. The technical assistance of $P$. Kayenbergh, G. Meulemans, and M. De Paep is gratefully acknowledged. We thank S. Raiguel and H. Op de Beeck for comments on a previous version of this manuscript, and R. Peeters for taking the structural magnetic resonance images.

*B.-E.V. and G.K. contributed equally to this work.

Correspondence should be addressed to Rufin Vogels, Laboratorium voor Neuro-en-Psychofysiologie, Katholiek Universiteit Leuven Medical School, Campus Gasthuisberg, 0\&N2, Herestraat 49, Bus 1021, BE-3000 Leuven, Belgium. E-mail: rufin.vogels@med.kuleuven.be.

D0I:10.1523/JNEUROSCI.3333-08.2008

Copyright $\odot 2008$ Society for Neuroscience $\quad 0270-6474 / 08 / 2810631-10 \$ 15.00 / 0$ with regard to higher cortical areas, little is known about the relationship of adaptation strength to test and adapter similarity. This is because previous studies of adaptation in higher cortical areas [e.g., inferior temporal (IT) cortex] have not systematically varied parameters of the adapter and test stimuli along a stimulus dimension (Miller et al., 1993) as has been done in investigations of early areas. In the present study, we wanted to test the underlying assumption of the fMR-A paradigm: the conjecture that the degree of adaptation scales with the similarity between adapter and test stimuli in higher cortical areas. We manipulated the size of successively presented shapes, allowing us to relate adaptation effects to the similarity between the adapter and test stimulus and to neural selectivity.

We recorded from neurons in both IT and prefrontal (PFC) cortical areas. We studied IT because previous monkey and human fMRI studies have used repetition suppression to infer invariances of ventral-stream neurons (Sawamura et al., 2005; Grill-Spector et al., 2006). Previous single-cell studies of PFC reported a variety of repetition effects ranging from suppression (Kubota et al., 1980; Rainer et al., 1999; Zaksas and Pasternak, 2006) to enhancement (Miller et al., 1996; Rainer et al., 1999) of the responses to a repeated stimulus. Furthermore, a monkey fMRI study showed adaptation effects in PFC similar to those in IT (Sawamura et al., 2005). Therefore, we also recorded from PFC, allowing a comparison of repetition effects, and of the relationship between physical stimulus similarity, neural selectivity, and adaptation strengths, in IT and PFC.

We used a same-different task in which animals judged 
whether two successively presented stimuli differed in shape or size. This task is similar to that of several human fMR-A studies in which it served to control for potential differences in attention between successive stimulus presentations (Epstein et al., 2003; Murray and Wojciulik, 2004; Kourtzi and Huberle, 2005; Krekelberg et al., 2005; Ganel et al., 2006; Murray et al., 2006; GilaieDotan and Malach, 2007). The values of the timing parameters of this task were chosen so as to resemble those of the rapid, eventrelated fMR-A studies as much as possible. Hence, our findings contribute to the understanding of results obtained using the fMR-A paradigm.

\section{Materials and Methods}

Subjects and recordings. Two rhesus monkeys (Macaca mulatta), YA and YE, served as subjects. All surgical procedures and animal care were in accordance with the guidelines of the Katholieke Universiteit Leuven Medical School and the European Communities Council Directive 86/ 609/EEC. The experiments were approved by the Ethical Committee for Animal Experiments of the Katholieke Universiteit Leuven.

For both IT and PFC recordings, a Narishige microdrive was mounted firmly on the recording chamber. The implantation of the plastic recording chambers was guided using preoperative anatomical MRI. For IT recordings, a tungsten microelectrode (Frederic Haer; 1-2 $\mathrm{M} \Omega$ measured in situ) was lowered through a guiding tube positioned in a grid (Christ Instruments) that was attached to the microdrive. The guiding tube served a twofold purpose: puncturing the dura and overlying tissue, and also guiding the electrode toward the recording location, which was quite distant with respect to the entrance point of the guide tube on the dura (we used the vertical approach toward IT). The PFC recordings were performed without a guiding tube so that the cortex directly underlying the dura would not be damaged by the guiding tube and could be recorded from. For these transdural PFC recordings, we drilled 2-4 mm craniotomies and recorded from the underlying cortex for as long as we were able to penetrate the dura with glass-coated tungsten electrodes (Ainsworth; impedance, $\sim 1 \mathrm{M} \Omega$ ). For both areas, the electrode signals were amplified and filtered using the same standard single-cell recording equipment. Single units were isolated on-line using the templatematching software Signal Processing Systems (SPS). The timing of the single units, the stimulus and behavioral events were stored with $1 \mathrm{~ms}$ resolution on a personal computer for later off-line analysis.

Between recording sessions, anatomical MRIs, using a grid with copper sulfate-filled capillary tubes inserted at selected recording positions, defined the region that we had recorded from. The IT recordings were from neurons in the lower bank of the superior temporal sulcus and the lateral convexity (area TE). Most responsive neurons were from the lower bank of the superior temporal sulcus. Based on the structural MRI scans, the IT recording positions ranged from $\sim 14$ to $22 \mathrm{~mm}$ anterior to the auditory meatus and from $\sim 19$ to $22 \mathrm{~mm}$ lateral from the midline. The PFC recording locations were anterior to the arcuate sulcus (area $8 \mathrm{Av}$ ) (Petrides and Pandya, 2002) and ventral to or in the posterior third of the principal sulcus (area 9/46) (Petrides and Pandya, 2002).

The position of one eye was recorded using the ISCAN eye movement monitoring system but not saved.

Stimuli. Stimuli were presented on a 21 inch display (viewing distance, $\sim 55 \mathrm{~cm}$ ). We used nine stimulus sets that each consisted of four different shapes in four different sizes. The four sizes ranged from $\sim 2$ to $16^{\circ}$ (in one octave steps). All stimuli were black shapes presented on a gray background. For a given size, the shapes of a given set had approximately the same area and luminance. See Figure 1 for an example of one set. All stimuli were centered on the middle of the screen and were familiar to the animals during recording.

Experimental procedure and task. The monkeys were trained to perform two sequential same-different tasks (see Fig. 1). In both tasks, they first fixated a fixation spot that was presented in the middle of the screen for $700 \mathrm{~ms}$. The first stimulus (S1) was presented for $306.6 \mathrm{~ms}$, followed by a delay of $506.6 \mathrm{~ms}$. After this delay, the second stimulus (S2) was presented for $306.6 \mathrm{~ms}$. During the presentation of the stimuli, the fixation spot was extinguished but the animal had to continue fixating. The second stimulus was followed by the presentation of two target points at $10^{\circ}$ eccentricity on either side of the fixation spot. The monkey had to make a saccade to the right or left target point in "same" and "different" trials, respectively. Only trials in which the saccades were directed toward one of the two target points were counted as valid trials and were scored as a correct or an error trial. The animal had to maintain its gaze in a fixation window $\left(\right.$ size,$\sim 2^{\circ}$ ) during the 700 -ms-long prestimulus fixation period, the stimulus presentations, and the delay period. Trials that did not meet this fixation criterion (i.e., aborted trials) were not analyzed further.

Whether a particular stimulus sequence was a same or different trial depended on the task. In the size task, the four different shapes of a set were presented at four different sizes. In the size task, S1 and S2 always had the same shape but could differ in size. Trials in which the sizes of S1 and S2 were identical were defined as same trials in the size task, whereas trials in which the sizes differed were defined as different trials. Thus, in the size task, the monkey had to judge whether or not a stimulus differed in size. In the shape task, the same stimuli were presented; however, on a given trial in this task, S1 and S2 could have either the same or different shapes and could have different sizes. Trials in which the shape was identical, regardless of a possible size difference, were defined as same trials in the shape task, whereas trials in which S1 and S2 differed in shape were defined as different trials. Thus, in the shape task, the monkey had to judge whether the two shapes were the same or different, independent of the size. In each task, the stimuli were presented pseudorandomly, obeying the following three constraints: (1) a particular stimulus should be presented equally often as S1 and S2, (2) the average frequency of each of the 16 stimuli was equal, and (3) the number of same and different trials should be equal. These constraints made certain that, to perform successfully, the monkey needed to compare S1 and S2.

The monkeys were extensively trained in these two tasks, using stimuli other than those used during the recordings. During the recordings, they performed the two tasks in alternating blocks of $\sim 180$ valid trials. Each block was preceded by 30 "instruction trials," in which the correct response, and therefore the task, was cued by presenting only the correct target point. These instruction trials were excluded from all analyses. The order of the two task blocks was counterbalanced across neurons.

Responsive neurons were searched while the monkeys were performing the tasks with the 16 stimuli of a set. In some sessions, several sets were tested successively to obtain a responsive neuron. The only selection criterion we used during the recordings was that the neuron should respond to at least one of the 16 stimuli of a set, as judged from inspection of the on-line peristimulus time histograms (PSTHs).

Analysis of neural data. The unnormalized response to a stimulus was defined as the number of spikes during an interval of $250 \mathrm{~ms}$, starting 50 $\mathrm{ms}$ after stimulus onset. As a measure of the baseline activity, we used the number of spikes in the $250 \mathrm{~ms}$ interval preceding $\mathrm{S} 1$ onset. If, for a given cell, a second block of a task was recorded, then these two blocks were combined in the analyses. To statistically test whether a neuron responded to one or more of the stimuli, we used ANOVA with "baseline" versus "stimulus period" as a within-trial factor and "stimulus" as between-trials factor. The statistical significance of the response to a particular stimulus was tested using Wilcoxon's matched-pairs tests comparing baseline and stimulus responses. Type I errors for statistical testing were set at $5 \%$.

The average size tuning of the population of neurons was computed by selecting, for each neuron, the shapes for which there was a significant mean S1 response (250 ms analysis window), and then sorting the combinations of shape and neuron as a function of the best size. Thus, we obtained four groups, each having a different preferred size. After normalizing the responses for each neuron by its maximal response over groups, the S1 responses were averaged for each preferred-size group, thus obtaining the average size tunings for the four different groups.

To obtain a quantitative measure of the degree to which the response of each neuron was modulated by size or shape, we computed size and shape modulation indices, respectively. These indices were computed for the responses to S1 only, taking into account all unaborted trials and regardless of the $\mathrm{S} 2$ stimulus. The size modulation index was computed for sizes of the same shape, taking only those shapes for which there was 
a significant $\mathrm{S} 1$ response. The shape modulation index was computed for shapes having the same size, taking only those sizes for which there was a significant $\mathrm{S} 1$ response. As a modulation index, we used the $\omega^{2}$ statistic (Kirk, 1968), which measures the proportion of response variance explained by size (shape) for a given shape (size). To obtain a reliable estimate of the response variance, computation of the $\omega^{2}$ was restricted to those sizes (shapes) for which at least four trials per stimulus were available. A larger $\omega^{2}$ indicates a greater size or shape modulation.

To quantify the strength of the adaptation effect, the mean response of a neuron to a particular stimulus presented as $\mathrm{S} 2$ was subtracted from the mean response to that same stimulus presented as $\mathrm{S} 1$. The response to the S2 presentation was computed as a function of the difference between S1 and S2 in a trial (i.e., S1-S2 sequence) (e.g., S1, size 1; S2, size 2). In this way, we obtain the repetition index, which is defined as follows:

$$
\text { Repetition index }(i, j)=\overline{\operatorname{Stim}(i) \_S 1}-\overline{\operatorname{Stim}(i) \_S 2(j)},
$$

where $\overline{\operatorname{Stim}(i) \_S 1}$ is the mean response to stimulus $i(1 \leq i \leq 16)$ presented as S1, and similarly $\overline{\operatorname{Stim}(i) \_S 2(j)}$ is the mean responses to stimulus $i$ presented as S2 for a particular S1-S2 sequence $j$. The possible $\mathrm{S} 1-\mathrm{S} 2$ sequences depended on the task. That is, S1 and S2 could only differ in size for the size task, whereas for the shape task the size and the shape of S1 and S2 could differ within a trial (see above). Hence, this index assesses for each S1-S2 sequence to what degree the response to a particular stimulus presented as S2 is affected by the preceding stimulus in a trial. The index will be zero if the response to a particular stimulus presented as $\mathrm{S} 2$ is equal to the response when that same stimulus was presented as S1. A positive value indicates that the response to a stimulus presented as S2 was smaller than when that same stimulus was presented as S1 (i.e., adaptation). Note that the responses that were used to compute the repetition indices could have come from different trials. For example, to compute $\overline{\operatorname{Stim}(i) \_S 1}$, we used all responses of a neuron to stimulus $i$ presented as the first stimulus (S1) in a trial, even when the second stimulus (S2) in a trial differed (e.g., in size or shape) from stimulus $i$. Similarly, to compute $\overline{\operatorname{Stim}(i) \_S 2(j)}$, we used all responses of a neuron to stimulus $i$ presented as the second stimulus in a particular S1-S2 sequence $j$, where $S 1$ could differ from stimulus $i$ depending on the particular S1-S2 sequence $j$. Repetition indices were computed for each neuron and task separately. The repetition index was computed for each stimulus for which there was a significant response, either as S1 or as S2, as tested by the Wilcoxon matched pairs test. As a result, $64 \%$ of the stimuli were selected to compute the repetition indices for the size and shape tasks for IT, whereas 62 and $60 \%$ of the stimuli were selected to compute the repetition indices for the size and shape tasks, respectively, in PFC.

The repetition indices were computed for the neural responses in three time windows: $250 \mathrm{~ms}$ starting $50 \mathrm{~ms}$ after stimulus onset, $125 \mathrm{~ms}$ starting $50 \mathrm{~ms}$ after stimulus onset, and $125 \mathrm{~ms}$ starting $175 \mathrm{~ms}$ after stimulus onset. The long $250 \mathrm{~ms}$ window examines the response during the stimulus presentation. The spiking activity during the initial $50 \mathrm{~ms}$ of the stimulus presentation was not examined, because this was not stimulus related (i.e., was shorter than the response latency of the neurons). The two $125 \mathrm{~ms}$ windows split the longer $250 \mathrm{~ms}$ window into two equal, nonoverlapping intervals, which allowed us to examine whether the repetition effects differed between the early and late parts of the response.

For the main analyses, the repetition indices were computed as a function of the relative size difference (in octaves) between S2 and the preceding $\mathrm{S} 1$ in a trial. For this reason, all repetition indices related to $\mathrm{S} 1-\mathrm{S} 2$ sequences with equal relative size differences between S1 and S2 were averaged and this was done for each of the possible size differences. In the main analysis, we assessed the effect of the size difference between $\mathrm{S} 1$ and S2 on the suppression when S1 and S2 had the same shape. In the latter analysis, we used S2 responses from only "same-shape" trials when determining the repetition effects as a function of the relative size difference between S1 and S2. Thus, all unaborted trials were used for the size task, whereas for the shape task approximately one-half of the trials were used to compute S2 responses [i.e., those in which S1 and S2 had the same shape (but could differ in size)]. The median number of trials per neuron used to compute the repetition indices for each relative size difference condition is shown in Table 1 for the size and shape tasks separately.
Table 1. Median number of same-shape trials per neuron to compute repetition indices, for each relative size difference between S1 and S2 (zero to three octaves; columns)

\begin{tabular}{llllll}
\hline \multirow{5}{*}{ Area } & Task & Octaves & & \\
\cline { 3 - 6 } & Zero & One & Two & Three \\
\hline IT & Size task & 69 & 35 & 22 & 12 \\
PFC & Size task & 65 & 34 & 23 & 10 \\
IT & Shape task & 18 & 29 & 12 & 12 \\
PFC & Shape task & 17 & 29 & 12 & 11 \\
\hline
\end{tabular}

Repetition indices were computed for unnormalized as well as normalized responses. For the latter, the responses of each neuron were normalized by the maximal mean response of the 16 stimulus conditions. The latter 16 responses were computed by averaging the responses to a particular stimulus when it appeared as S1 and as S2. This normalization ensured that each neuron, even those that responded weakly to any of the 16 stimuli, contributed equally to the population response. The normalization was performed for each task separately. The repetition indices were computed from these normalized responses using the formula given above.

Modeling blood oxygen level-dependent response. We convolved the unsmoothed IT and PFC population PSTHs of the size task with the hemodynamic response function as defined by Boynton et al. (1996) to predict the blood oxygen level-dependent (BOLD) response obtained in a fast event-related fMR-A paradigm. To ensure that the differences in the BOLD response were related only to the differences in the S2 response and were not contaminated by small response fluctuations between the four size difference conditions that preceded S2 onset, the responses before S2 onset were equalized in the four conditions. This was done using the time-varying mean of the spike rate in the time interval including the $300 \mathrm{~ms}$ preceding S1, the S1 presentation, and the subsequent delay; this mean was computed across the four conditions. When the original $\mathrm{S} 1$ and delay responses were used, the difference in the predicted BOLD between zero and one to three octaves was even more pronounced for IT, whereas differences between the one, two, and three octave conditions were somewhat smaller. The hemodynamic response function was modeled as a gamma function [formula 3 in the study by Boynton et al. (1996)] with parameters $n=3, \tau=1.4$, and delay of 2.4 s. Other values of $\tau$ and delay produced highly similar size difference effects. The values for each of the size difference conditions were normalized with respect to the same-size condition (zero octave) data (minimum, 0 ; maximum, 1). For the PFC prediction, we used the responses up to $\$ 2$ offset to avoid any contamination of the predicted BOLD response by the third periresponse peak in the PSTH. Using the full PSTH produced a stronger difference between the zero and one to three octave conditions (as expected from the difference in neural responses of these conditions at this periresponse period) but no systematic, graded increase in BOLD response with size difference. We excluded the third response peak because it may depend on the behavioral paradigm and operant or on eye movement control during this late periresponse phase. The responses before S2 onset were equalized in the four conditions in the same way as the IT responses. When the original S1 and delay responses were used, results were similar, and in this case also, the predicted BOLD show no graded increase with size difference.

\section{Results}

We recorded the responses to different-sized shapes in 176 single IT and 126 PFC neurons in two macaques while the animals were performing same-different tasks. Each neuron was tested with a set of 16 stimuli that consisted of four different shapes at four different sizes (range, three octaves). During the recordings, the monkeys performed one of two tasks: In the size task, the stimuli of a given trial had the same shape and could differ only in size, whereas in the shape task, the successive stimuli could differ in both size and shape. In the latter task, the animals were required to make a size-invariant shape judgment (i.e., same or different 
shape), whereas in the former task, they simply made a size judgment (i.e., same or different size). The required behavioral response to a size difference of the same shape was opposite in the two tasks (Fig. 1), allowing a dissociation between sizecontingent adaptation effects and behavioral responses. The two tasks were presented in different blocks.

\section{Behavioral data}

We analyzed the performance of each animal in the two tasks using the behavioral data recorded during the neuronal tests. During the IT recordings, monkey YA's performance levels were highly similar in the two tasks (84 and $85 \%$ correct in the size and shape task, respectively), whereas monkey YE performed somewhat better in the size $(87 \%)$ than in the shape task $(83 \%)$. Both animals performed well above chance in each task and for each size difference. For the one octave size differences, the animals recognized the shape with an average performance of $88 \%$ correct. The size invariance of shape recognition was not complete: the performance in the shape task decreased as a function of the size difference in both animals. However, even for the three octave size differences, shape recognition averaged 69\% correct, which is well above chance level. Performance averaged $79 \%$ correct in the size task for the one octave size difference, indicating that the animals were able to discriminate shapes differing twofold in size when instructed to do so.

During the prefrontal recording sessions, the performance level averaged $89 \%$ correct in each of the two monkeys. This is $\sim 5 \%$ greater than during the IT recording sessions. This slightly better performance in the prefrontal experiments than in the preceding IT sessions is probably a practice effect. As for the IT sessions, monkey YA's performance was similar in the two tasks (shape task, 88\%; size task, 90\%), whereas the other animal was more proficient in the size $(92 \%)$ than in the shape $(86 \%)$ task. As during the IT recordings, the performance in the shape task decreased as a function of the relative size difference. Thus, the relative percentages correct for each monkey over the various tasks and conditions were similar during the IT and prefrontal recordings.

\section{Neuronal data}

We determined the size selectivity of the neurons using their responses to the S1 presentations when the animals were performing the same-different tasks. Across our population of neurons, single IT and PFC neurons were well tuned for size (Fig. $2 A, D)$. As expected, the IT and PFC responses decreased gradually as the stimulus size diverged from the preferred size. The effect of size on the neural response was on average greater in PFC

$700 \mathrm{Msec}$
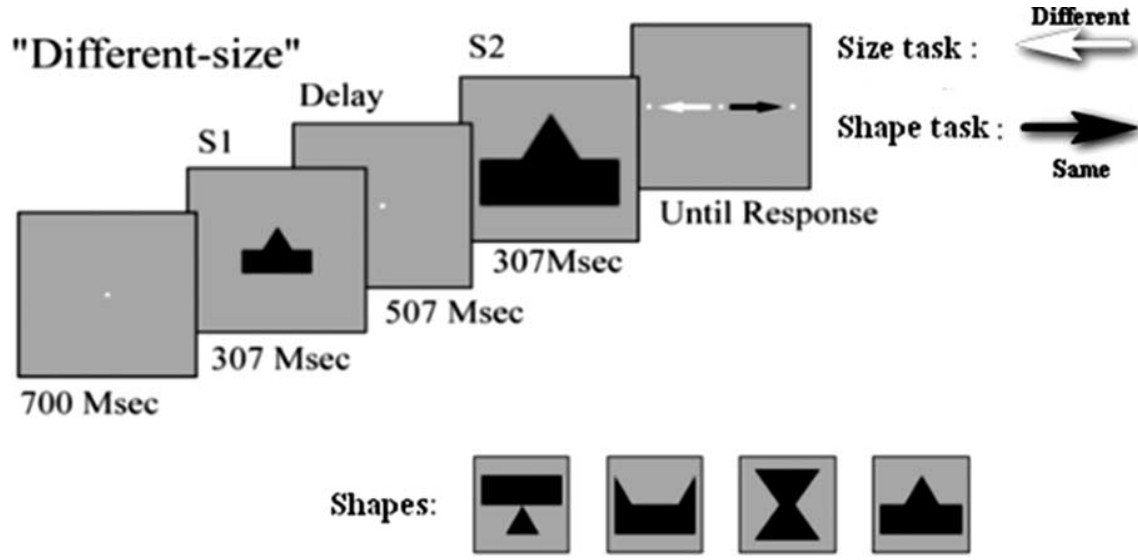

Figure 1. Event sequence in a different-size trial and shapes for an example stimulus set. The monkey was required to make a leftward saccade after presentation of $\$ 2$ when the stimulus sizes differed in the size task (different response), whereas for the same stimulus sequence, the animal had to make a rightward saccade (same response) in the shape task, because the stimuli themselves had the same shape. Thus, the correct response for the same stimulus sequence depended on the task that the monkey was instructed to perform (for additional details, see Materials and Methods). Below are shown four shapes from one stimulus set.
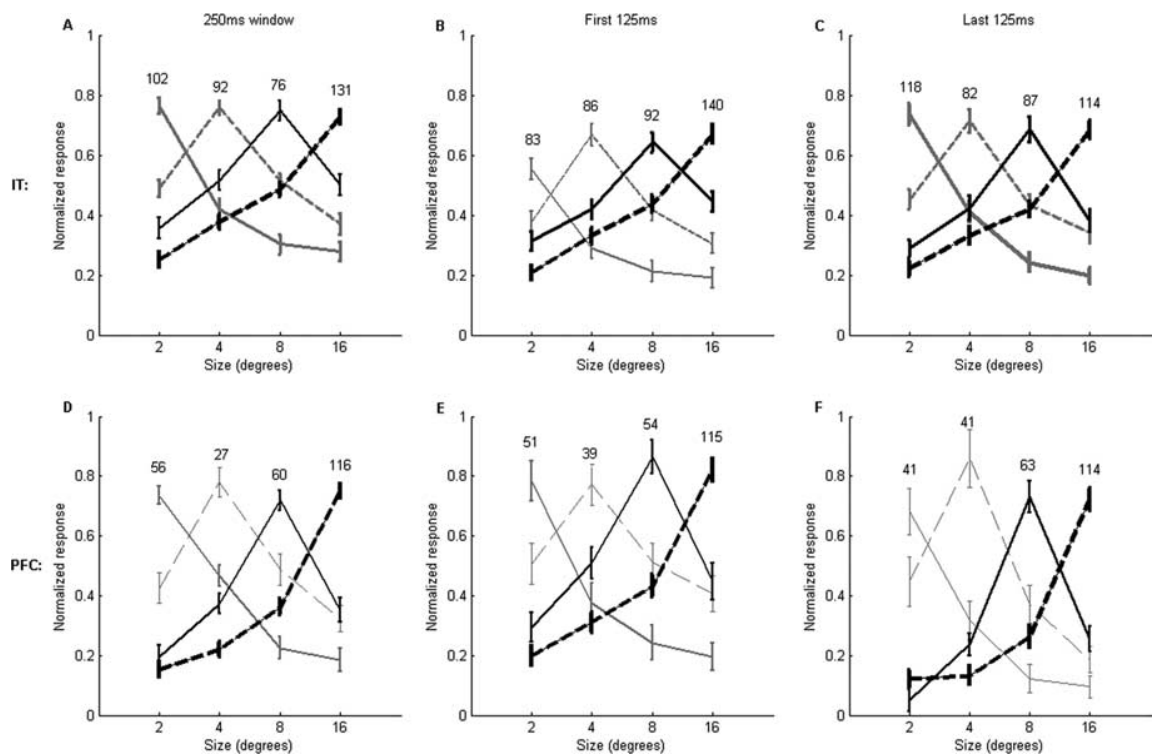

Figure 2. Size selectivity in IT and PFC. $\boldsymbol{A}-\boldsymbol{C}$, IT neurons. $\boldsymbol{D}-\boldsymbol{F}, \mathrm{PFC}$ neurons. The normalized size-tuning curves are plotted using the full $250 \mathrm{~ms}$ analysis window $(\boldsymbol{A}, \boldsymbol{D})$, and the first $125 \mathrm{~ms}(\boldsymbol{B}, \boldsymbol{E})$ and second $125 \mathrm{~ms}(\boldsymbol{C}, \boldsymbol{F})$ analysis windows (size task only). Firing rates to $\mathrm{S} 1$ of each neuron were normalized with respect to the maximum firing rate of the 16 stimulus conditions (based on the S1 response). The latter were computed using the full $250 \mathrm{~ms}$ window (otherwise potential differences in response level between the first and second analysis windows would be obscured). The mean neuronal responses for the different sizes of each shape with a significant response were sorted according to the preferred size and then averaged. Error bars indicate SEM. Different line styles are used to indicate tuning curves with different preferred sizes. The number on top of the preferred size of each tuning curve specifies the number of cases that were used to compute that tuning curve.

\section{Table 2. Size and shape modulation in IT and PFC}

\begin{tabular}{|c|c|c|c|c|c|}
\hline Area & Task & Median $\omega^{2}$; size & $n$ & Median $\omega^{2}$; shape & $n$ \\
\hline IT & Size task & 0.10 & 401 & 0.25 & 459 \\
\hline PFC & Size task & 0.17 & 259 & 0.18 & 257 \\
\hline IT & Shape task & 0.12 & 368 & 0.24 & 411 \\
\hline PFC & Shape task & 0.20 & 216 & 0.20 & 220 \\
\hline
\end{tabular}

than in IT ( $p<0.001$, Wilcoxon's rank sum test), as can be seen by considering the median $\omega^{2}$ size modulation indices computed on the S1 responses (Table 2). Within each area, the degree to which the response was modulated by size was similar for the two tasks (Table 2) ( $p>0.05$, Wilcoxon's rank sum test). 


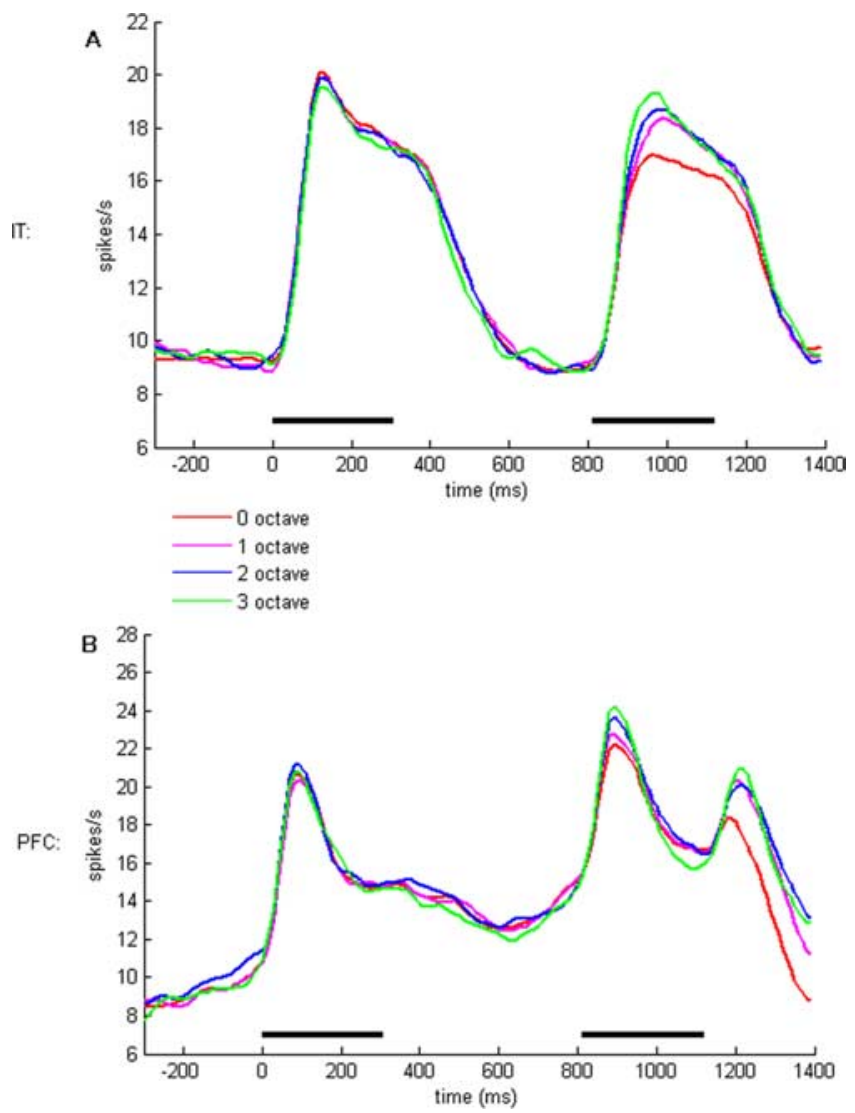

Figure 3. $\quad \boldsymbol{A}, \boldsymbol{B}$, Population PSTHs of the responses in IT and PFC in the size task as a function of the size difference. Trials were sorted as a function of the size differences (in octaves) between S1 and S2. Responses were first averaged per condition and per neuron, and these means were then averaged across neurons. Horizontal bars indicate stimulus presentations. All neurons responding to any of the stimuli were averaged [IT $(\boldsymbol{A}), N=155 ; \operatorname{PFC}(\boldsymbol{B}), N=106]$. The population responses were smoothed using a Gaussian kernel $(\sigma=20 \mathrm{~ms})$.

Given that both areas showed clear size selectivity, we can address the question of whether these areas will show sizecontingent adaptation. This issue was examined by analyzing the degree of adaptation as a function of the size differences between S1 and S2. First, we will describe the results for IT followed by those for PFC.

\section{Size-contingent repetition effects in IT}

As expected (Baylis and Rolls, 1987; Miller et al., 1991, 1993; Vogels et al., 1995; Ringo, 1996; Jiang et al., 2006; Sawamura et al., 2006), IT responses were reduced when the same-size stimulus was repeated. In a first analysis to demonstrate the effect of a size difference between S1 and S2, we used only trials from the size task. In the latter task, S1 and S2 always consisted of the same shape and thus more trials per size difference were presented than in the shape task, in which in only one-half of the trials the successive stimuli had the same shape. Figure $3 A$ plots the average population responses to $\mathrm{S} 1$ and S2 as a function of the S1-S2 size difference within a trial of the size task. It is clear that the strength of the adaptation effect diminished gradually with increasing size differences between S1 and S2.

To quantify the strength of the adaptation effect, the response of a neuron to a particular stimulus presented as S2 was subtracted from that to the same stimulus presented as $S 1$ (repetition index) (see Materials and Methods). Positive and negative repetition indices indicate decreased and enhanced responses to S2 with respect to $S 1$, respectively. The repetition indices were com-
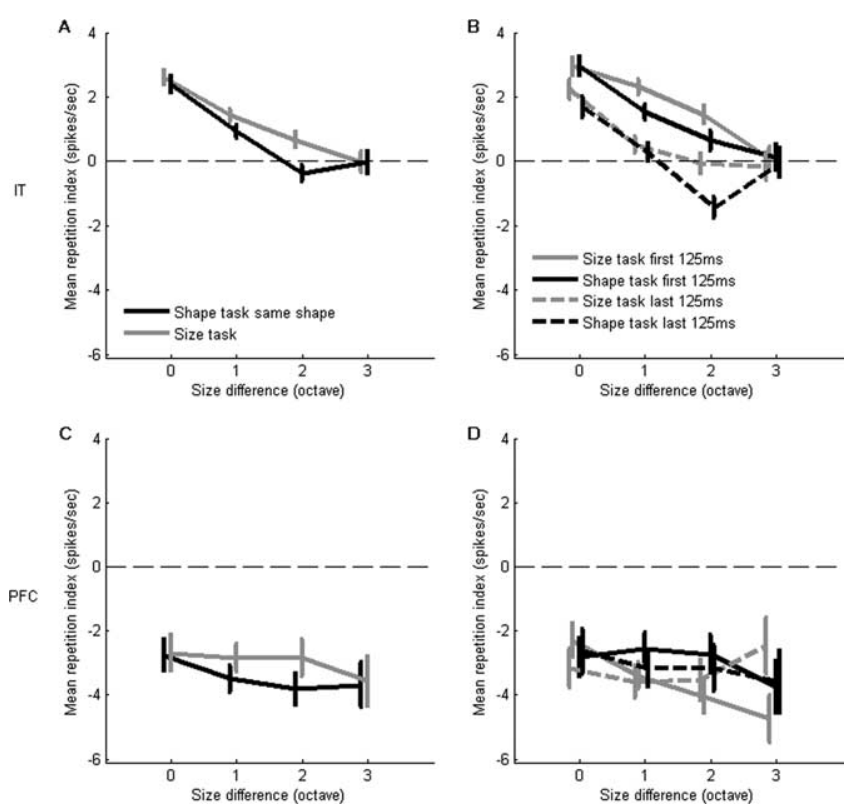

Figure 4. $\quad \boldsymbol{A}-\boldsymbol{D}$, Size-contingent adaptation in IT and PFC. Mean difference between the response to a stimulus presented as $S 1$ and the response to that same stimulus presented as $S 2$ in IT and PFC. Trials were sorted as a function of the difference in the sizes of $\mathrm{S} 1$ and $\mathrm{S} 2$ in the size and shape tasks. Positive and negative repetition indices indicate decreased and enhanced responses to $\$ 2$, respectively. The responses were computed in three analysis windows: $250 \mathrm{~ms}$ starting $50 \mathrm{~ms}$ after stimulus onset $(\boldsymbol{A}, \boldsymbol{C})$ and the first and second half of this window $(\boldsymbol{B}, \boldsymbol{D})$.

puted as a function of the size difference between S2 and the preceding S1 of that trial. This analysis was restricted to those stimuli and neurons for which there were statistically significant responses when the stimulus was presented as S1 or as S2 (see Materials and Methods). In these initial analyses, the responses were computed using an analysis window of $250 \mathrm{~ms}$ that started $50 \mathrm{~ms}$ after stimulus onset. The mean repetition indices decreased as a function of the S1-S2 size difference (Fig. 4A). ANOVA showed that the effect of this size difference on the repetition indices was highly significant $(p<0.001)$. Furthermore, the gradual decrease of the strength of adaptation with increasing size differences was statistically significant in each animal $(p<0.001)$ (supplemental Fig. S1, available at www.jneurosci.org as supplemental material). We also computed repetition indices using normalized responses, and the results obtained in this manner were similar to those reported above for the unnormalized responses: the repetition indices decreased significantly (ANOVA, $p<0.0005$ ) with increasing size differences in the size task when the full $250 \mathrm{~ms}$ analysis window was considered (supplemental Fig. S2, available at www.jneurosci.org as supplemental material).

An unexpected finding was that the size dependency of the adaptation was more graded in the first half of the IT response than in its second half (Fig. $3 A$ ). To quantify this observation, we computed the repetition indices separately for the first and second $125 \mathrm{~ms}$ of the $250 \mathrm{~ms}$ analysis window (Fig. $4 \mathrm{~B}$ ). The interaction between size difference and analysis window proved to be significant (ANOVA; $p<0.01$ ). In contrast to the first $125 \mathrm{~ms}$ window, there was no significant effect of size difference on the repetition indices when we considered only the one, two, and three octave size differences for the second $125 \mathrm{~ms}$ window (ANOVA: second half, $p>0.05$; first half, $p<0.0005$ ). Thus, the effect of size difference on the IT adaptation is less graded in the second than in the first half of the S2 response. Similar results 
were obtained for normalized responses: The interaction between size difference and analysis window for the normalized responses was significant (ANOVA, $p<0.05$ ), and ANOVAs performed on the repetition indices of the one to three size difference conditions of the size task produced a significant main effect of size difference for the first $125 \mathrm{~ms}$ analysis window $(p<$ $0.0005)$ but not for the second $125 \mathrm{~ms}$ window $(p>0.05)$ (see supplemental information and supplemental Fig. S2, available at www.jneurosci.org as supplemental material).

The data shown in Figure 4 are repetition indices averaged across stimuli and neurons. When the responses of each individual neuron were averaged across stimuli instead, similar effects could be demonstrated (supplemental Fig. S3, available at www. jneurosci.org as supplemental material). For this analysis, we fitted an orthogonal regression line to the mean response of each neuron to a stimulus when presented as S1 and S2. The analysis was repeated for each relative size difference between S1 and S2 within a trial and for each of the three time windows. The mean S1 and S2 responses of each neuron were computed using the same responses as the ones that were used to compute the repetition indices, but now these responses were averaged across stimuli within a neuron. When considering the full analysis window, we observed a significant suppression of the S2 responses for the zero and one octave size differences ( $p<0.05 ; z$ test for a difference in the slope compared with the first bisector). Using the first $125 \mathrm{~ms}$ window, we found significant suppression of the S2 responses for the zero, one, and two octave size differences. In contrast, a significant suppression only for the zero size difference was noted when the second $125 \mathrm{~ms}$ window was used. The degree of adaptation, as measured by the slope of the regression line for the S1 and S2 responses, decreased monotonically with increasing size difference for the first, but not second, $125 \mathrm{~ms}$ window (supplemental Fig. S3, available at www.jneurosci.org as supplemental material).

A trivial explanation for the size difference having a more weakly graded effect in the second, late analysis window is that IT neurons could simply possess a correspondingly weaker size tuning in that part of their response. However, as shown in Figure 2, $B$ and $C$, the graded size tuning was equally present in both the first and second $125 \mathrm{~ms}$ analysis windows. Thus, the weak graded effect of size difference on the adaptation in the second window is not some trivial result of a simple difference in size tuning within these two analysis windows.

The mean responses to the S1 stimuli, computed using the same S1 stimuli as those for the repetition indices, did not differ significantly among the four size difference groups for either the full $250 \mathrm{~ms}$, the early $125 \mathrm{~ms}$, or the late $125 \mathrm{~ms}$ windows (ANOVA, $p>0.05$ ). Thus, the observed effect of size difference on the repetition indices does not result from any differences in the strengths of the $S 1$ responses between the four size difference groups, but is instead a genuine adaptation effect.

One could hypothesize that the less graded neural response in the second than in the first part of the neural response to S2 is related to the disparate behavioral responses in the same and different trials of the size task. If such were the case, then one would expect an effect of task on the repetition indices because the "different-size-same-shape" trials required opposite behavioral responses in the two tasks. This possibility was examined in a series of two-way ANOVAs, with S1-S2 size difference and task as factors, on the normalized and unnormalized repetition indices for the $250 \mathrm{~ms}$ and the two $125 \mathrm{~ms}$ analysis windows separately. Note that, for a proper comparison, the indices in both tasks were computed using only same-shape trials. The $p$ values of
Table 3. Task effects in IT

\begin{tabular}{llll}
\hline Analysis window & Effect & Unnormalized & Normalized \\
\hline $250 \mathrm{~ms}$ & Task & $\mathbf{0 . 0 1 5}$ & $\mathbf{0 . 0 0 4}$ \\
& Size difference & $\mathbf{0 . 0 0 0}$ & $\mathbf{0 . 0 0 0}$ \\
\multirow{2}{*}{ First $125 \mathrm{~ms}$} & Interaction & 0.226 & 0.291 \\
& Task & 0.073 & $\mathbf{0 . 0 1 8}$ \\
& Size difference & $\mathbf{0 . 0 0 0}$ & $\mathbf{0 . 0 0 0}$ \\
Second $125 \mathrm{~ms}$ & Interaction & 0.280 & 0.408 \\
& Task & $\mathbf{0 . 0 3 0}$ & $\mathbf{0 . 0 4 1}$ \\
& Size difference & $\mathbf{0 . 0 0 0}$ & $\mathbf{0 . 0 0 0}$ \\
& Interaction & 0.168 & 0.105 \\
\hline
\end{tabular}

Significant $p$ values $(p<0.05$, two-way ANOVA) are shown in bold.

all effects are listed in Table 3, with significant effects indicated in bold. We observed no significant interactions of S1-S2 size difference and task. There were some significant main effects of task on the repetition indices. Note that these main effects of task cannot be related to potential behavioral response-related effects, because the latter require an interaction of the factors task and size difference (the behavioral response depends on the size difference in the size task but not in the shape task).

Additional analyses of the data from the shape task showed that the mean response to S2 decreased systematically as a function of the S1-S2 size difference within the first $125 \mathrm{~ms}$ analysis window (mean repetition indices shown in Fig. $4 \mathrm{~B}$; mean responses per neuron shown in supplemental Fig. S4, available at www.jneurosci.org as supplemental material). However, for the second $125 \mathrm{~ms}$ window, such size dependence was less clear and erratic: the two octave size difference produced enhancement of the responses to S2, whereas no significant difference in S2 response was present for the one and three octave size differences. Thus, as for the size task, the systematic decrease of repetition suppression with increasing size differences was present only within the first part of the response (see also supplemental Fig. S7, available at www.jneurosci.org as supplemental material, which shows the population PSTHs for the shape task).

In the above analyses, the responses in all valid (error and correct) trials were averaged. Supplemental Figure S8 (available at www.jneurosci.org as supplemental material) shows the mean repetition indices computed using the responses in only the correct same-shape trials. The effects obtained using correct trials only were virtually identical with those observed using all sameshape trials. Again, no significant interactions between the effects of size difference and task were observed when correct trials only were used. Note that the monkeys executed opposite responses for the one to three octave differences in these same-shape trials: same responses in the shape task and different responses in the size task. These analyses demonstrate that the adaptation effects, particularly in the later part of the response in IT, are not related to the impending same versus different behavioral responses.

Very similar results were obtained when the responses to the preferred size for each neuron were excluded (supplemental Fig. S9, available at www.jneurosci.org as supplemental material). Hence the suppression effects were not specific to the preferred size.

\section{Size-contingent repetition effects in PFC}

Subsequent recordings in the PFC of the same animals showed response patterns that differed from those seen in IT. In agreement with previous recordings in PFC using similar tasks, the PFC population showed anticipatory-related climbing activity before S1 and S2 onset, and stronger delay activity than IT (Fuster and Alexander, 1971; Miller et al., 1996; Rainer et al., 1999) (Fig. 

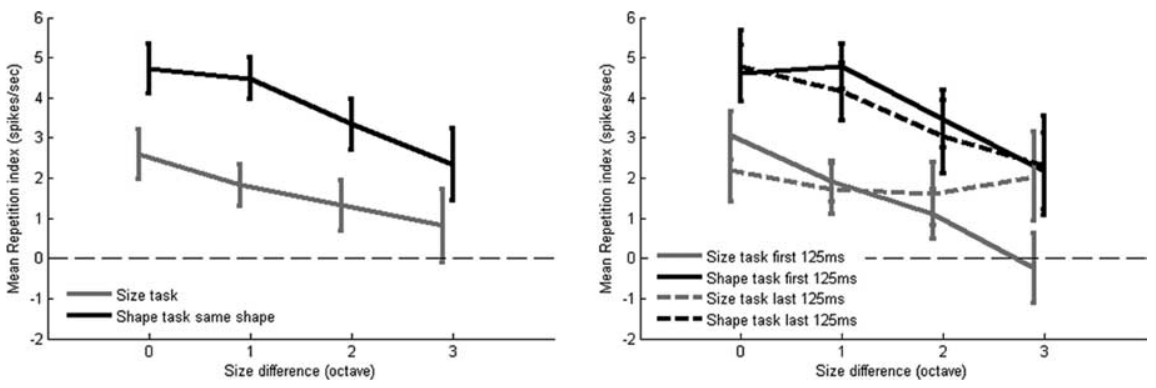

Figure 5. Size-contingent adaptation in PFC for putative stimulus-driven responses. The analyses and conventions were the same as those of Figure 4, but the $\$ 1$ and $\$ 2$ responses were corrected for baseline and delay activity, respectively (for details, see Results).

3B). Also, several PFC neurons responded during the behavioral response phase, which can be seen as the third peak in the population PSTHs of Figure $3 B$.

We used the same analyses as that for IT to assess repetition effects in PFC and their relationship to size differences between S1 and S2. When computing S1 and S2 responses as for IT, we found that, on average, PFC neurons showed an enhanced response to the $\mathrm{S} 2$ presentation when the stimulus was repeated (Figs. 3B, 4C). This enhancement was not affected by the size difference between S1 and S2 when the full $250 \mathrm{~ms}$ analysis window (Fig. $4 C$ ) was considered (ANOVA, $p>0.05$ ). However, there was a significantly (ANOVA, $p<0.05$ ) increased enhancement in the first half of the response with greater size differences (Fig. 4D). This effect was present only for the size task, although the interaction between size difference and task was not significant. Furthermore, we did not find this effect when normalized responses (see supplemental information, available at www. jneurosci.org as supplemental material) were used, and, contrary to what was observed in IT, the interaction between size difference and time window was not significant (ANOVA, $p>0.05$ ). Thus, overall, the systematic dependence of the $\mathrm{S} 2$ responses on the S1-S2 size difference was much smaller in PFC compared with IT, using the same analyses. As for IT, very similar results were obtained when we excluded the responses to the preferred size for each neuron (supplemental Fig. S9, available at www. jneurosci.org as supplemental material), and thus the suppression effects were not specific to the preferred size.

As noted above and shown in Figure 3, PFC neurons showed strong delay activity. It could be argued that the stimulus-driven response overlies this delay activity. Following that logic, we isolated this putative stimulus-driven response to S2 by subtracting the activity in a $250 \mathrm{~ms}$ interval of the delay period immediately preceding S2 from the activity to S2. To compute the stimulusdriven $\mathrm{S} 1$ response, we subtracted the baseline activity in a $250 \mathrm{~ms}$ period before S1 onset from the activity to S1. Not surprisingly, given the strong delay activity, the average putative stimulusdriven S2 responses were smaller than those to S1: the mean repetition indices computed from the stimulus-driven responses using the $250 \mathrm{~ms}$ analysis windows were positive (Fig. 5), indicating repetition suppression. Although the repetition indices decreased with increasing size differences for both tasks, this effect was not significant in either task (ANOVAs, $p>0.05$ ). Combining the data of the two tasks produced a significant effect of size difference (ANOVA, $p=0.04$ ) on the repetition indices. When the repetition indices were computed using the (baseline- and delay activity-corrected) first half $(125 \mathrm{~ms})$ of the responses, the effect of size difference was significant for the size task (ANOVA, $p<0.01$ ) but not for the shape task (ANOVA, $p>0.05$ ). No significant effects of size difference were present for the repetition indices computed from the second half of the responses. In none of these analyses did we observe a significant interaction between task and size difference. Also, the interaction of the factors analysis window (first vs second half) and size difference was not significant. These analyses indicate weak effects of size difference on the putative stimulus-driven responses of PFC neurons.

\section{Shape-contingent repetition effects in IT and PFC}

Both IT and PFC demonstrated shape selectivity. The degree of modulation of the neural response by shape, as assessed by the $\omega^{2}$ measure for S1 responses (Table 2), was significantly larger in IT than in PFC ( $p<0.005$, Wilcoxon's rank sum test) but did not differ significantly between tasks (Table 2$)(p>0.05$, Wilcoxon's rank sum test). Because we did not manipulate shape similarity in the current study, we will only briefly describe the shapecontingent repetition effects. As can be seen in Figure 6, responses to $\mathrm{S} 2$ in different-shape trials were enhanced with respect to S1 and S2 in same-shape trials. For these analyses, responses of only the same-size trials were used. The enhancement of S2 responses in different-shape trials was present in IT and PFC. This enhancement was statistically significant ( $t$ test; mean different from zero, $p<0.05$ ). Additional analyses using the $250 \mathrm{~ms}$ analysis window showed a significant difference between the repetition indices computed for the same- and different-shape trials in IT (ANOVA, $p<0.001$ ) but not in PFC (ANOVA, $p>0.05$ ). There was no effect of size difference on the repetition indices for the different-shape trials in either region (ANOVA, $p>0.05$ ) (supplemental Figs. S1, S8, available at www.jneurosci.org as supplemental material).

\section{Simulating BOLD response}

The changes in the stimulus dependency of adaptation effects over the course of the response that we have observed in IT can affect the sluggish BOLD response and can thus affect inferences concerning selectivity that are based on adaptation in rapid, event-related fMR-A (Kourtzi and Kanwisher, 2000) [assuming that the BOLD response indeed correlates with the neural response (Logothetis, 2003)]. Following Mukamel et al. (2005), Sawamura et al. (2006), and Privman et al. (2007), we estimated the BOLD response by convolving the population response in the size task (Fig. 3) with a standard canonical hemodynamic response function (Boynton et al., 1996). The estimated BOLD response in IT differentiated the zero and one octave condition well, but less so for the one, two, and three octave conditions (Fig. $7 A$ ). Thus, the estimated BOLD size adaptation effect is more strongly nonlinear than the size tuning of the neural responses (Fig. 2A).

The estimated BOLD responses for PFC in the size task showed smaller, less systematic size difference-dependent repetition effects (Fig. $7 B$ ) compared with the size tuning of the same neuronal population (Fig. 2D). Thus, from these estimated BOLD responses, one would infer little or no systematic size tuning in PFC, which is certainly not the case (Fig. 2D). 

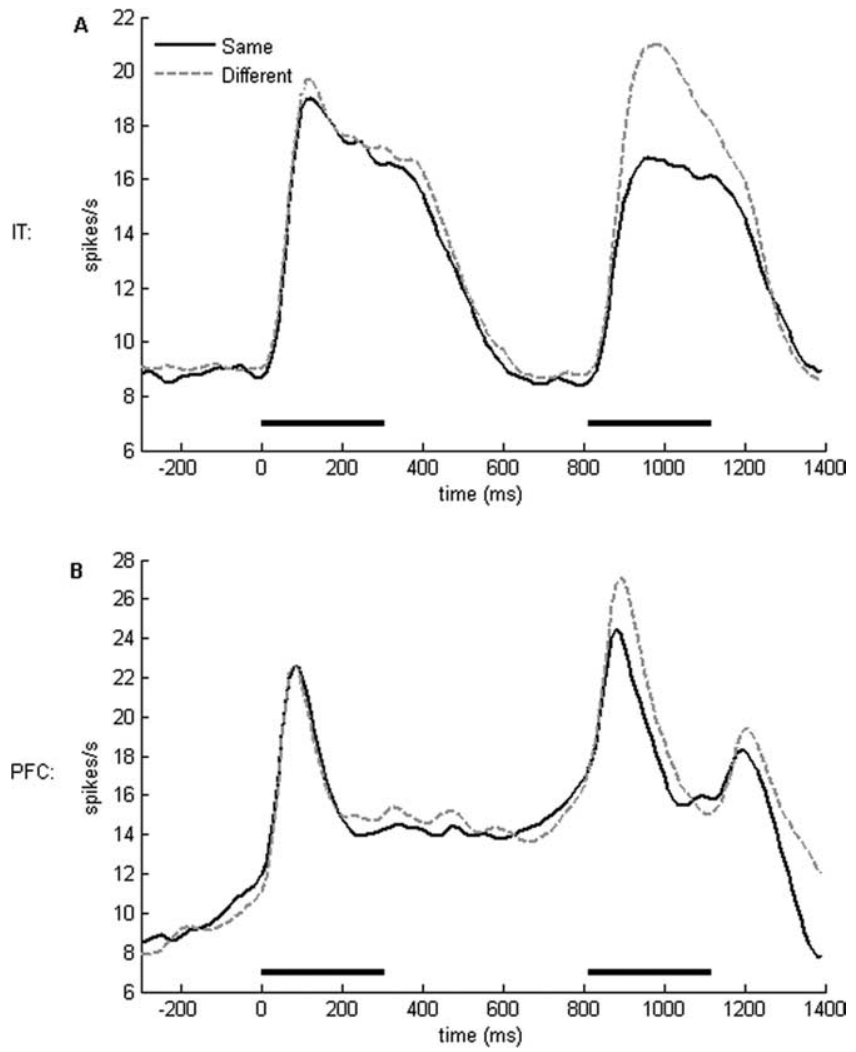

Figure 6. Population PSTHs of the responses in IT $(\boldsymbol{A})$ and PFC $(\boldsymbol{B})$ in the shape task for same-shape and different-shape trials. The $\mathrm{S} 1$ and $\mathrm{S} 2$ stimuli had identical sizes. Responses were first averaged per condition and per neuron, and these means were then averaged across neurons. The same conventions as in Figure 3 apply.
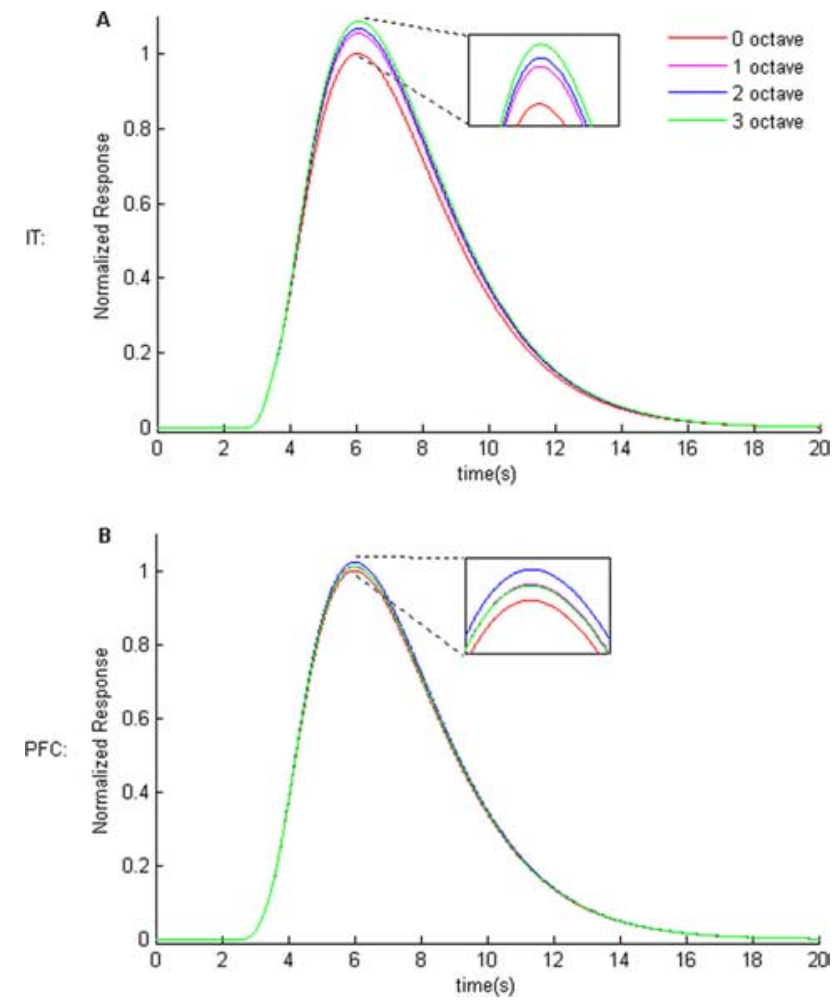

Figure 7. Estimated normalized BOLD responses for the IT $(\boldsymbol{A})$ and PFC $(\boldsymbol{B})$ population responses in the size task, sorted according to the size difference (in octaves; see legend) between S1 and $\mathrm{S} 2$.

\section{Discussion}

We have shown that the degree of adaptation in IT, and to a much lesser extent in PFC, depends systematically on stimulus size differences and thus on the similarity between the adapter and test stimuli. The size difference effect became less graded in the later part of the IT response. In contrast, despite the obvious size tuning in prefrontal neurons, these cells did not demonstrate a clear relationship between stimulus similarity and the size of the repetition effect when the latter was computed on the gross response. Together, these findings are highly relevant for the frequently used fMR-A paradigm. This paradigm is based on the assumed link between feature tuning and tuning in adaptation when stimuli along the relevant feature dimension are presented in succession. We show that this relationship can exist in higher cortical areas, such as IT, where fMR-A is often used.

\section{Size selectivity}

In contrast to the size-dependent adaptation effects in the present study, Lueschow et al. (1999) reported size-invariant adaptation in the IT cortex of monkeys performing a delayed matching-tosample task. Several differences between the two studies may explain these discrepant results. First, Lueschow et al. recorded in ventral IT/perirhinal cortex, whereas the neurons of the present study were from dorsal IT/superior temporal sulcus. Second, we used a wider range of sizes than did Lueschow et al. (1999) (one octave). It is possible that their neurons would have shown significant size-dependent adaptation for larger relative size differences. Third, unlike the study by Lueschow et al., our animals were trained in the size task, and thus the size-dependent adaptation effect may be induced by this training. However, Sawamura et al. (2005) found size-dependent adaptation of the fMRI activation in monkey IT when the animals did not have to discriminate size. The results of the latter monkey fMRI study suggest that, even when animals are not trained to discriminate size, adaptation of shape responses in IT can be size dependent.

We found that PFC neurons were well tuned for size. It cannot be excluded that part of the size selectivity in PFC results from training in the size discrimination task. Indeed, training to discriminate visual images can enhance the selectivity of PFC neurons for those images (Rainer and Miller, 2000).

\section{Time course of stimulus selective repetition effects}

Our data demonstrate that the size-dependent adaptation response becomes more "categorical" (same vs different size) in the later part of the IT response. Because of this time-dependent effect in combination with the relative long duration of the IT response (compared with stimulus duration), the predicted BOLD response will be more sensitive to a change in size per se than to the magnitude of the size change. One fMRI study using timing parameters similar to those of the present study reported that face viewpoint-sensitive adaptation in the human visual cortex was independent of the amount of rotation (Fang et al., 2007), which fits our BOLD prediction.

\section{Effect of task on stimulus selectivity}

We performed extensive data analyses comparing the responses and degree of size and shape selectivity between the two tasks (data not shown), but failed to find consistent task effects on these parameters in IT and PFC. The lack of task-related effects on the IT stimulus selectivity agrees with recent reports by Suzuki et al. (2006) and Lehky and Tanaka (2007), but contrasts with that by Koida and Komatsu (2007). However, in the latter study, 
the two tasks differed in several respects such as categorization versus fine discrimination and fixed stimulus-response mapping versus matching to sample, whereas in our study the subjects were required to perform the identical "same-different" task while changing the relevant stimulus dimension between tasks. Thus, the smaller dissimilarity between tasks in the present study might explain our lack of any task effect.

It is possible that the animals solved the two tasks by changing the decision boundary in a high-dimensional space defined by the different shapes and sizes. Following this reasoning, the decision boundary in the size task would have been shifted toward smaller size differences. Note that such a mechanism does not assume changes in selectivity between the tasks: the task determines the position of the decision boundary without affecting the sensitivity for the stimulus dimensions. Hence this proposed mechanism can explain why we did not observe consistent changes in size or shape selectivity between the tasks in IT and PFC.

\section{Effect of task on repetition effects}

We observed no systematic effects of the task on the adaptation effects for the same-shape trials in IT neurons. This agrees with several rapid, event-related $\mathrm{AMR}-\mathrm{A}$ human studies that reported approximately similar adaptation effects in different tasks (Murray and Wojciulik, 2004; Krekelberg et al., 2005; Xu et al., 2007), at least when the subject is attending the stimulus (Murray and Wojciulik, 2004). For example, the latter human fMRI study found similar view-dependent BOLD adaptation in LOC when subjects performed view-invariant object recognition and when they judged the view of an object (Murray and Wojciulik, 2004). More recently, Xu et al. (2007) compared BOLD adaptation when subjects were performing two sorts of same-different tasks, one in which they had to recognize a scene regardless of changes in view, and one in which they had to discriminate changes in the images or the view of the same scene. In agreement with our results, no effect of task on BOLD adaptation was observed in the ventral visual cortex.

There was a hint of a task-related effect in PFC, consisting of a trend for a greater size-dependent enhancement of the S2 responses in the size task than in the shape task within the first part of the response. This weak task-related effect might be the result of our search strategy: because we were interested in stimulusrelated activity, we searched for neurons showing stimulusrelated responses. Hence we may have missed PFC neurons that code for the task but did not respond to the stimuli (e.g., units showing stronger stimulus-unrelated activity in one task compared with the other) (Asaad et al., 2000; Wallis et al., 2001; Wallis and Miller, 2003; Mansouri et al., 2006).

\section{Comparison of IT and PFC repetition effects}

Anatomical studies indicate that IT projects to the PFC region that we recorded from (Ungerleider et al., 1989; Webster et al., 1994). Interestingly, the effects of size and shape of S1 on the S2 population response originated earlier in PFC than in IT (Fig. 3), suggesting that at least the early size-dependent effects in PFC do not originate in IT. Dorsolateral PFC, within and close to the principal sulcus, has connections with intraparietal sulcal areas (Petrides and Pandya, 1984). Therefore it is possible that the rapidly responding PFC neurons receive direct or indirect input from intraparietal neurons, which can have short stimulusselective response latencies. One caveat here is that the PFC recordings were performed after the IT recordings and that the faster response in PFC compared with IT might be a practice effect.
Despite the similar size tunings of neurons in IT and PFC, we found markedly different stimulus repetition effects in the gross neuronal responses of these two areas. Repetition effects were both different in sign (repetition suppression and enhancement for IT and PFC, respectively) and in its relationship with stimulus similarity. The weaker size-contingent adaptation effects in PFC compared with IT could lead to the erroneous inference that PFC shows a smaller size selectivity than IT, which was not the case. One possible cause of such marked interregional differences in repetition effects is that task (i.e., execution of a same-different task) and stimulus repetition interact differently in IT and PFC. Thus, the enhancement of S2 responses in PFC may depend on the execution of the same-different tasks. Such a task-related effect might explain the discrepancy between the present finding, of weak size-contingent adaptation in PFC single neurons, and size-contingent adaptation of the fMRI signal in PFC in a previous monkey fMRI study (Sawamura et al., 2005) in which the animals were not engaged in discriminating the stimuli. Because we used one interstimulus interval and stimulus duration in the present study, we cannot exclude that the weak adaptation effects in PFC are partially attributable to the presentation parameters.

The results of the analyses whereby we took into account the activity preceding the stimulus presentations suggest that task and repetition effects indeed interact in PFC. These putative stimulus-driven PFC responses showed repetition suppression and, albeit weak, size-dependent repetition effects. Note that this analysis assumes that delay and stimulus-driven components of the response during stimulus presentation are additive, which is uncertain. More importantly, fMRI activations do not subtract delay activity for the delay durations used in the rapid-event designs, and would thus correspond to the gross neural activity. BOLD would therefore be expected to show size-related repetition effects in PFC differing from those in IT, despite similar size tunings for the responses.

In a previous study, we showed that the response selectivity of IT neurons can be less than the stimulus selectivity of the adaptation effect (Sawamura et al., 2006), indicating that fMR-A results should be interpreted with caution. The present study demonstrates that stimulus-selective adaptation effects can be region and time dependent, implying that interregional comparisons of fMR-A-based inferences of stimulus selectivity should be made cautiously.

\section{References}

Asaad WF, Rainer G, Miller EK (2000) Task-specific neural activity in the primate prefrontal cortex. J Neurophysiol 84:451-459.

Baylis GC, Rolls ET (1987) Responses of neurons in the inferior temporal cortex in short term and serial recognition memory tasks. Exp Brain Res 65:614-622.

Boynton GM, Engel SA, Glover GH, Heeger DJ (1996) Linear systems analysis of functional magnetic resonance imaging in human V1. J Neurosci 16:4207-4221.

Epstein R, Graham KS, Downing PE (2003) Viewpoint-specific scene representations in human parahippocampal cortex. Neuron 37:865-876.

Fang F, Murray SO, He S (2007) Duration-dependent fMRI adaptation and distributed viewer-centered face representation in human visual cortex. Cereb Cortex 101:447-454.

Fuster JM, Alexander GE (1971) Neuron activity related to short-term memory. Science 173:652-654.

Ganel T, Gonzalez CL, Valyear KF, Culham JC, Goodale MA, Köhler S (2006) The relationship between fMRI adaptation and repetition priming. Neuroimage 32:1432-1440.

Gilaie-Dotan S, Malach R (2007) Sub-exemplar shape tuning in human face-related areas. Cereb Cortex 17:325-338.

Grill-Spector K, Henson R, Martin A (2006) Repetition and the brain: neural models of stimulus-specific effects. Trends Cogn Sci 10:14-23. 
Jiang X, Rosen E, Zeffiro T, Vanmeter J, Blanz V, Riesenhuber M (2006) Evaluation of a shape-based model of human face discrimination using FMRI and behavioral techniques. Neuron 50:159-172.

Kirk RE (1968) Experimental design: procedures for the behavioral sciences. Belmont, CA: Brooks/Cole Publishing.

Kohn A (2007) Visual adaptation: physiology, mechanisms, and functional benefits. J Neurophysiol 97:3155-3164.

Koida K, Komatsu H (2007) Effects of task demands on the responses of color-selective neurons in the inferior temporal cortex. Nat Neurosci 10:108-116.

Kourtzi Z, Huberle E (2005) Spatiotemporal characteristics of form analysis in the human visual cortex revealed by rapid event-related fMRI adaptation. Neuroimage 28:440-452.

Kourtzi Z, Kanwisher N (2000) Cortical regions involved in perceiving object shape. J Neurosci 20:3310-3318.

Krekelberg B, Vatakis A, Kourtzi Z (2005) Implied motion from form in the human visual cortex. J Neurophysiol 94:4373-4386.

Krekelberg B, Boynton GM, van Wezel RJA (2006) Adaptation: from single cells to BOLD signals. Trends Neurosci 29:250-256.

Kubota K, Tonoike M, Mikami A (1980) Neuronal activity in the monkey dorsolateral prefrontal cortex during a discrimination task with delay. Brain Res 183:29-42.

Lehky SR, Tanaka KJ (2007) Enhancement of object representations in primate perirhinal cortex during a visual working-memory task. J Neurophysiol 97:1298-1310.

Logothetis NK (2003) The underpinnings of the BOLD functional magnetic resonance imaging signal. J Neurosci 23:3963-3971.

Lueschow A, Miller EK, Desimone R (1999) Inferior temporal mechanisms for invariant object recognition. Cereb Cortex 5:523-531.

Mansouri FA, Matsumoto K, Tanaka KJ (2006) Prefrontal cell activities related to monkeys' success and failure in adapting to rule changes in a Wisconsin Card Sorting Test analog. J Neurosci 26:2745-2756.

Miller EK, Gochin PM, Gross CG (1991) Habituation-like decrease in the responses of neurons in inferior temporal cortex of the macaque. Vis Neurosci 7:357-362.

Miller EK, Li L, Desimone RJ (1993) Activity of neurons in anterior inferior temporal cortex during a short-term memory task. J Neurosci 13:1460-1478.

Miller EK, Erickson CA, Desimone R (1996) Neural mechanisms of visual working memory in prefrontal cortex of the macaque. J Neurosci 16:5154-5167.

Mukamel R, Gelbard H, Arieli A, Hasson U, Fried I, Malach R (2005) Coupling between neuronal firing, field potentials, and fMRI in human auditory cortex. Science 309:951-954.

Murray SO, Wojciulik E (2004) Attention increases neural selectivity in the human lateral occipital complex. Nat Neurosci 7:70-74.

Murray SO, Olman CA, Kersten D (2006) Spatially specific FMRI repetition effects in human visual cortex. J Neurophysiol 95:2439-2445.
Naccache L, Dehaene S (2001) The priming method: imaging unconscious repetition priming reveals an abstract representation of number in the parietal lobes. Cereb Cortex 11:966-974.

Petrides M, Pandya DN (1984) Projections to the frontal cortex from the posterior parietal region in the rhesus monkey. J Comp Neurol 228:105-116.

Petrides M, Pandya DN (2002) Comparative cytoarchitectonic analysis of the human and the macaque ventrolateral prefrontal cortex and corticocortical connection patterns in the monkey. Eur J Neurosci 16:291-310.

Privman E, Nir Y, Kramer U, Kipervasser S, Andelman F, Neufeld MY, Mukamel R, Yeshurun Y, Fried I, Malach R (2007) Enhanced category tuning revealed by intracranial electroencephalograms in high-order human visual areas. J Neurosci 27:6234-6242.

Rainer G, Miller EK (2000) Effects of visual experience on the representation of objects in the prefrontal cortex. Neuron 27:179-189.

Rainer G, Rao SC, Miller EK (1999) Prospective coding for objects in primate prefrontal cortex. J Neurosci 19:5493-5505.

Ringo JL (1996) Stimulus specific adaptation in inferior temporal and medial temporal cortex of the monkey. Behav Brain Res 76:191-197.

Sawamura H, Georgieva S, Vogels R, Vanduffel W, Orban GA (2005) Using functional magnetic resonance imaging to assess adaptation and size invariance of shape processing by humans and monkeys. J Neurosci 25:4294-4306.

Sawamura H, Orban GA, Vogels R (2006) Selectivity of neuronal adaptation does not match response selectivity: a single-cell study of the FMRI adaptation paradigm. Neuron 49:307-318.

Suzuki W, Matsumoto K, Tanaka KJ (2006) Neuronal responses to object images in the macaque inferotemporal cortex at different stimulus discrimination levels. J Neurosci 26:10524-10535.

Ungerleider LG, Gaffan D, Pelak VS (1989) Projections from inferior temporal cortex to prefrontal cortex via the uncinate fascicle in rhesus monkeys. Exp Brain Res 76:473-484.

Vogels R, Sary G, Orban GA (1995) How task-related are the responses of inferior temporal neurons? Vis Neurosci 12:207-214.

Wallis JD, Miller EK (2003) From rule to response: neuronal processes in the premotor and prefrontal cortex. J Neurophysiol 90:1790-1806.

Wallis JD, Anderson KC, Miller EK (2001) Single neurons in prefrontal cortex encode abstract rules. Nature 411:953-956.

Webster MJ, Bachevalier J, Ungerleider LG (1994) Connections of inferior temporal areas TEO and TE with parietal and frontal cortex in macaque monkeys. Cereb Cortex 4:470-483.

Xu Y, Turk-Browne NB, Chun MM (2007) Dissociating task performance from $\mathrm{fMRI}$ repetition attenuation in ventral visual cortex. J Neurosci 27:5981-5985.

Zaksas D, Pasternak T (2006) Directional signals in the prefrontal cortex and in area MT during a working memory for visual motion task. J Neurosci 26:11726-11742. 